\title{
Exploring the moderating effect of social intelligence on the relationship between entrepreneurial decision-making strategy and SME sustainable performance
}

\author{
Dayang Hasliza Muhd Yusuf ${ }^{1 *}$, Mohd Salleh Hj. Din², and Muhamad Shahar Jusoh ${ }^{3}$ \\ ${ }^{123}$ University Malaysia Perlis (UniMAP), School of Technopreneurship and Business Innovation, 01000 Kangar, Perlis, Malaysia
}

\begin{abstract}
The study reveals that causation, rather than effectuation, decision-making strategy is a more significant predictor of sustainable performance of SMEs. However, social intelligence was not found to be a significant moderator of entrepreneurial decision-making-sustainable performance relationship. The study uses data from a survey among 91 technology-based SMEs (TBS) in Malaysia and employs structural equation modelling techniques for data analysis. A new instrument to measure all three variables of entrepreneurial decision-making strategy, social intelligence, and venture performance is proposed based on adoption and adaptation of existing validated scales available in literature.
\end{abstract}

Key words: Effectuation, causation, entrepreneurial decision-making, social intelligence, SME, sustainable performance

\section{Introduction}

The study examined the moderating influence of social intelligence (SI) over the entrepreneurial decisionmaking strategy (EDM) and sustainable performance (SP) relationship of technology-based SMEs (TBS) in Malaysia. SP is defined as the extent to which an SME's achievement of the socio-efficiency objectives over its competitors [1]. While effectuation, rather than causation, has been argued as the more prominent thought process in uncertain environments [2]. Prior studies have established that due to the high uncertainty in starting a new venture, entrepreneurs need to be responsive to changes. Therefore, effectuation or nonpredictive thinking is argued to make a company nimble and adaptable to the unpredictable shifts in the marketplace. At the same time, the importance of SI as the ability to understand, managed people and behave correctly in any social setting and has been associated with individual achievement in leadership, sales, and marketing [3]. However, whether SI can further improve the EDM-SP relationship need to be further explored.

\subsection{Entrepreneurial Decision-Making Strategy}

EDM has been argued to be within the realm of causative (or predictive) thinking and effectuation. Causation is planning-oriented based on entrepreneur's assessment of opportunities, whereas effectuation is along the stream of arguments that opportunities can be created rather than discovered $[4,2]$. On the other hand, causation consists of strategies that combine available means with unanticipated contingencies to construct a series of stakeholder commitment [2].

\subsection{Social Intelligence}

Unlike general or cognitive intelligence, SI focuses on the ability to get along well with others and interacting successfully in any social setting [5]. Various authors look at SI from various perspectives of cognitive, behavioural, and psychometric [6,7]. Authors offer varied definitions and measurement of the construct such as "the individual's fund of knowledge about the social world" [8], and how people perceive, react, and interact with the social world in which they live [6], and suggested the working definition of SI is suggested as "the ability to understand other people and how they will react to different social situations" [7]. SI has been suggested to be important for performance of selling and marketing activities [8,9], and has influence over individual achievement. As among the challenge faced by TBS in Malaysia is marketing performance, there is a need to explore the effect of SI on the EDM-SP relationship. Therefore, this study aims to examine the EDM approach of entrepreneurs, and SI level of the entrepreneurs on the performance of TBS. The resourcebased view (RBV) is adopted as the underpinning theory in our investigation.

\subsection{Sustainable Performance}

There is limited convergence among researchers on the definition and the measurement of organizational performance $[10,11]$. For TBS, performance definition and measurement becomes more complex, and large firms, generic performance measures cannot be used for TBS [12]. Authors have also argued that the range of

\footnotetext{
*Corresponding author: dayanghasliza@unimap.edu.my
} 
venture performance in entrepreneurship research need to be broadened to include the achievement of economic, environmental and social value creation objectives [1].

\section{Study Framework and Development of Hypotheses}

Prior studies have established the positive and significant relationship between the decision-making strategy and venture performance. However, the findings on the relationship between effectuation and venture performance has also not been consistent [2]. Research has also pursued the idea that social ability is relatively important for entrepreneurs, however little empirical evidence has supported the idea [9]. Thus, the researcher proposes that a new moderating variable of SI be introduced to explain the inconsistent findings. Figure1 depicts the study framework and research hypothesis developed.

\subsection{Population and Sample}

The population of the proposed study are owners of TBS registered with several agencies responsible for developing SMEs. As of 31st December 2013, there are a total 5,301,378 of businesses registered with SSM [13]. In terms of representation, SMEs percentage to total business establishments dropped from $99.2 \%$ in 2003 to $97.3 \%$ in 2010 [14].

Time, resources, and non-availability of data and contact information of the whole population understudy are among the factors which makes it impossible to collect data from the whole population. Using sample which is representative of the population would be more logical. The sample of the study are incubatees companies of several incubators and technology parks in Malaysia.

\subsection{The Research Instrument}

Figure 1 Research framework depicting research hypothesis

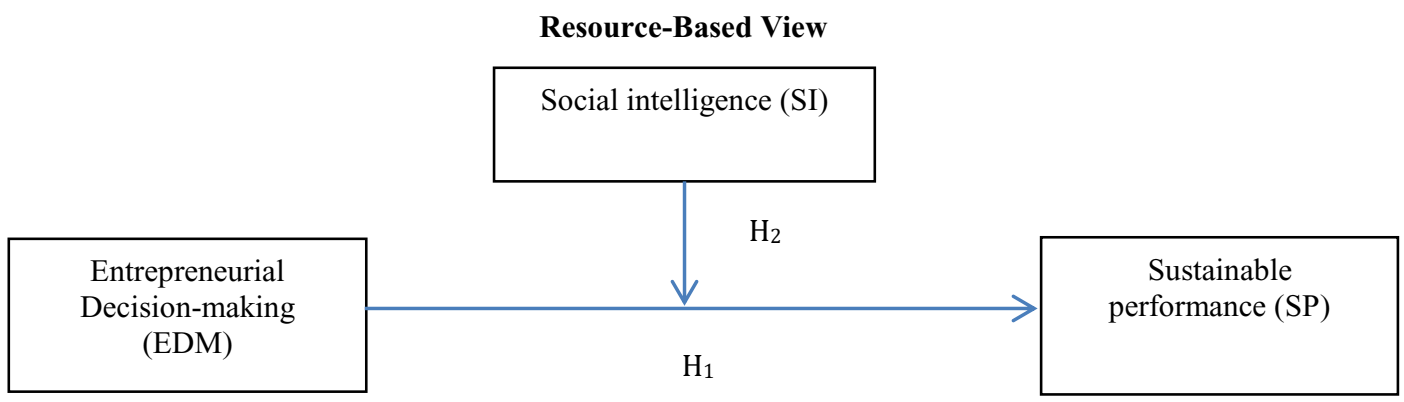

The expected relationship between all the variables, namely EDM, SI, and SP, used in developing the hypothesis for this study are as follows:

\section{$\mathrm{H}_{1}$ : EDM is positively related to $\mathrm{SP}$}

$\mathrm{H}_{2}$ : Upon introduction of SI, the strength and direction of the formerly positive significant relationship between EDM and SP relationship will change.

\subsection{Research Design}

Data collection was carried out via a survey using questionnaires. The questionnaire was constructed with adaptation and adoption of existing instruments to measure the level EDM, SI and SP in decision making involving creation / discovery and exploitation of opportunities. Prior to data collection, a pre-test of the instrument developed was conducted to measure the validity and reliability test of the instrument. Data was analysed using the multivariate statistical techniques. The individual entrepreneur is the unit of analysis.
The questionnaire consists of four sections, namely, 1) demographic, 2) entrepreneurial decision-making, 3) social intelligence, and 4) sustainable performance.

In the beginning, 14 items represent the EDM construct (seven items measure causation while the other seven items measure effectuation). However, four items measuring effectuation were dropped due to low scores for reliability. While the scale for SI was adapted from the 3-factor, 21-item Tromso Social Intelligence Scale (TSIS) [7]. However, upon pilot test of the instrument, only 11 items were found to have the reliability criteria in representing the SI construct. On the other hand, SP is measured by 11 items measuring economic, social, and environmental performance adapted from existing studies. Total remaining items in the questionnaire is 32 .

\section{Findings and Discussion}

The analysis involves looking at the effect on the direct relationship between EDM and SP $(\mathrm{DM} \rightarrow \mathrm{SP})$, when a third variable, SI, is introduced in the model. The model tested is as per Figure 1. Discussion for the indirect effect of SI on EDM and SP $(\mathrm{DM} \rightarrow \mathrm{SP})$ relationship was conducted starting with measurement model assessment, followed by goodness of fit assessment, and lastly with structural model assessment in subsequent topics. 


\subsection{Measurement Model Assessment}

Based on the PLS algorithm report generated, the values for the assessment criteria can be derived. Table 1, Table 2 , and Table 3 provide the values to be used for measurement model assessment of the study. Upon running the PLS-Algorithm function, three items measuring effectuation, and four items measuring SI were further dropped due to the low loadings of the items $(<0.6)$. The remaining items were 25 , of which seven items measure causation decision-making strategy, seven items for SI, and 11 items measures SP. Based on Table 1, the values of Cronbach's alpha for all variables are more than the recommended threshold of 0.8 [15], while the composite reliability (CR) values lie between 0.6 and 0.95 . Thus, the all the items assigned to the EDM, SI, and SP constructs in the study, are found to have high internal consistency reliability [16].

Table 1. Internal consistency reliability assessment results

\begin{tabular}{ccccc}
\hline Constructs Items & $\begin{array}{c}\text { Composite } \\
\text { Reliability } \\
\text { (CR) }\end{array}$ & $\begin{array}{c}\text { Cronbach's } \\
\text { Alpha }\end{array}$ & $\begin{array}{c}\text { Internal } \\
\text { consistency } \\
\text { reliability } \boldsymbol{\alpha} \\
>\mathbf{0 . 8} \text { and } \\
\mathbf{0 . 6 0}<\mathbf{C R}< \\
\mathbf{0 . 9 5}\end{array}$ \\
\hline EDM & 7 & 0.95 & 0.94 & YES \\
SI & 7 & 0.92 & 0.90 & YES \\
SP & 11 & 0.95 & 0.94 & YES \\
\hline
\end{tabular}

$\overline{\mathrm{EDM}}=$ Entrepreneurial decision-making strategy; $\mathrm{SI}=$ social intelligence; $\mathrm{SP}=$ sustainable performance

Next is the convergent validity test using examination of indicator reliability, and the average variance extracted (AVE) of all the constructs. Table 2 summarise the results of convergent validity tests based on AVE and factor loadings of each items. Items with loading values below 0.4 were deleted while remaining items with loading values below 0.708 were examined to see their effect on the construct's AVE score [17]. The values of factor loadings for the remaining 25 items lies above the threshold value of 0.708 as recommended by [18] indicating that all the items have much in common with other items within the same construct.

Table 2 Convergent validity based on item loadings and AVE for $S I \rightarrow D M \rightarrow S P$

\begin{tabular}{|c|c|c|c|c|c|}
\hline Constructs & Item & Loadings & AVE & $\begin{array}{l}\text { AVE } \\
>0.5\end{array}$ & $\begin{array}{c}\text { Loadings } \\
>0.708\end{array}$ \\
\hline \multirow{7}{*}{$\begin{array}{l}\text { Entrepreneu- } \\
\text { rial decision- } \\
\text { making } \\
\text { strategy } \\
(\text { EDM) }\end{array}$} & CA01 & 0.851 & 0.719 & YES & YES \\
\hline & $\mathrm{CA} 02$ & 0.912 & & & \\
\hline & CA03 & 0.833 & & & \\
\hline & CA04 & 0.826 & & & \\
\hline & CA05 & 0.826 & & & \\
\hline & CA06 & 0.815 & & & \\
\hline & CA07 & 0.816 & & & \\
\hline \multirow{7}{*}{$\begin{array}{c}\text { Social } \\
\text { intelligence } \\
\text { (SI) }\end{array}$} & SI01 & 0.832 & 0.633 & YES & YES \\
\hline & $\mathrm{SI02}$ & 0.798 & & & \\
\hline & SI03 & 0.812 & & & \\
\hline & SI04 & 0.803 & & & \\
\hline & SI05 & 0.771 & & & \\
\hline & SI06 & 0.785 & & & \\
\hline & SI07 & 0.768 & & & \\
\hline \multirow{3}{*}{$\begin{array}{l}\text { Sustainable } \\
\text { performance } \\
\text { (SP) }\end{array}$} & SP01 & 0.785 & 0.642 & YES & YES \\
\hline & SP02 & 0.768 & & & \\
\hline & SP03 & 0.758 & & & \\
\hline
\end{tabular}

$\begin{array}{ll}\text { SP04 } & 0.813 \\ \text { SP05 } & 0.822 \\ \text { SP06 } & 0.744 \\ \text { SP07 } & 0.856 \\ \text { SP08 } & 0.838 \\ \text { SP09 } & 0.826 \\ \text { SP10 } & 0.811 \\ \text { SP11 } & 0.815\end{array}$

$\mathrm{EDM}=$ Entrepreneurial decision-making strategy; $\mathrm{SI}=$ social intelligence; $\mathrm{SP}=$ sustainable performance

While for the convergent validity assessment based on the AVE criterion, based on Table 2, the values of AVE for DM, SI and SP constructs are more than the recommended value of 0.5 [17]. Thus, EDM shared $71.9 \%$, while SI shared $63.3 \%$, and SP shared $64.2 \%$ of variance with the assigned items. In summary, the convergent validity tests were fulfilled by the study model. The next assessment is the discriminant validity which is conducted by comparing the cross loadings of item associated with the EDM, SI, and SP against the associated values of loadings of the same items on other constructs. All the item loadings are found to be highest on each construct the items are supposed to measure. Thus, the convergent validity requirement on cross loadings examination is met. While for the evaluation based on the Fornell-Larcker criterion, Table 3 indicates that the square root values of AVE for all constructs are higher than the associated cross-loading figures, thus, the discriminant validity requirements are satisfied at both the item and construct level. The difference between loadings of items assigned to the DM construct are also more than 0.1 of the loadings on SP and vice versa [19, $20]$.

Table 3 Fornell-Larcker criterion evaluation for SI*DM $\rightarrow$ SP relationship

\begin{tabular}{ccccc}
\hline & AVE & DM & SI & SP \\
\hline DM & 0.719 & $\mathbf{0 . 8 4 8}$ & & \\
SI & 0.633 & 0.420 & $\mathbf{0 . 7 9 6}$ & \\
SP & 0.642 & 0.379 & 0.509 & $\mathbf{0 . 8 0 1}$ \\
\hline * Diagonal elements and in bold are square roots of AVE.
\end{tabular}

These results indicate that the indicators represent the assigned construct, and the extent to which all the constructs of DM, SI, and SP are truly distinct from each other. Lastly is the HTMT criterion which is the ratio of correlations within the constructs to correlations between the constructs $[21,22]$. Based on Table 4, the HTMT ratio values are 0.654 , and 0.536 , which are below 0.85 [23], indicating that the discriminant validity is ascertained for the moderation effect of SI on DM $\rightarrow$ SP relationship.

Table 4. HTMT criterion for SI*DM $\rightarrow$ SP

\begin{tabular}{llll}
\hline & \multicolumn{1}{c}{ DM } & \multicolumn{1}{c}{ SI } & SP \\
\hline & 0.654 & 0.536 & \\
& CI 0.90 & CI 0.90 & \\
SP & $(0.055,0.334)$ & $(0.241,0.563)$ & \\
\hline
\end{tabular}

Thus, for the indirect (moderating) effect of SI on EDM, and SP $(\mathrm{DM} \rightarrow \mathrm{SP})$ relationship, all the 
measurement model assessment criteria of internal consistency reliability, convergent validity, and discriminant validity were met by the items and constructs of EDM, SI, and SP. Table 5 summarise all the results of the measurement model assessment conducted on the constructs and items of the study.

Table 5 Summary of results for measurement model assessment for $\mathrm{SI} * \mathrm{DM} \rightarrow \mathrm{SP}$

\begin{tabular}{|c|c|c|c|}
\hline Type of test & $\begin{array}{c}\text { Evaluation } \\
\text { Criteria }\end{array}$ & Requirements & Results \\
\hline $\begin{array}{l}\text { Internal } \\
\text { consistency } \\
\text { reliability }\end{array}$ & $\begin{array}{l}\text { Cronbach's } \\
\text { alpha }(\alpha) \\
\text { Composite } \\
\text { Reliability } \\
(\mathrm{CR})\end{array}$ & $0.60<\mathrm{CR}<0.95$ & $\begin{array}{l}\text { Met for } \\
\text { all items } \\
\text { Met for } \\
\text { all } \\
\text { constructs }\end{array}$ \\
\hline $\begin{array}{ll}\text { Convergent } \\
\text { validity }\end{array}$ & $\begin{array}{l}\text { Indicator } \\
\text { reliability } \\
\text { Average } \\
\text { Variance } \\
\text { Extracted } \\
\text { (AVE) }\end{array}$ & $\begin{array}{l}\text { Outer loadings }> \\
0.5 \\
\text { AVE }>0.50\end{array}$ & $\begin{array}{l}\text { Met for } \\
\text { all items. } \\
\text { Met by all } \\
\text { constructs }\end{array}$ \\
\hline \multirow[t]{3}{*}{$\begin{array}{l}\text { Discrimi- } \\
\text { nant validity }\end{array}$} & $\begin{array}{l}\text { Cross } \\
\text { loadings of } \\
\text { indicators }\end{array}$ & $\begin{array}{l}\text { Indicator should } \\
\text { have the highest } \\
\text { loadings on the } \\
\text { assigned } \\
\text { construct } \\
\text { compared to its } \\
\text { loadings on other } \\
\text { constructs. }\end{array}$ & $\begin{array}{l}\text { Fully met } \\
\text { by all } \\
\text { items }\end{array}$ \\
\hline & $\begin{array}{l}\text { Fornell- } \\
\text { Larcker } \\
\text { Criterion } \\
(1981)\end{array}$ & $\begin{array}{l}\text { Square root of } \\
\text { AVE }>\text { latent } \\
\text { variable } \\
\text { correlations }\end{array}$ & $\begin{array}{l}\text { Fully met } \\
\text { for all } \\
\text { constructs }\end{array}$ \\
\hline & $\begin{array}{l}\text { HTMT } \\
\text { criterion }\end{array}$ & $\begin{array}{l}\text { HTMT }<0.85 \\
\text { Confidence } \\
\text { interval does not } \\
\text { have value of } 1 .\end{array}$ & $\begin{array}{l}\text { Met for } \\
\text { all } \\
\text { constructs }\end{array}$ \\
\hline
\end{tabular}

\subsection{Goodness of Fit (GoF) for Moderated ET $\rightarrow S P$ relationship}

This study will adopt the two assessment criteria, namely, SRMR and NFI assessment methods for the study model fit examination. Based on Table 6, the SRMR value is less than 0.10 which is the recommended threshold [24]. While the NFI value is 0.748 which is lower than the recommended value of at least $0.90[25$, 22]. Thus, the DM $\rightarrow$ SP model meets the model fit criteria for SRMR but not the NFI criteria. The next step is structural model assessment of the SI on DM $\rightarrow \mathrm{SP}$ relationship, which is discussed in the following subtopic.

\begin{tabular}{lcc}
\hline & Saturated Model & Estimated Model \\
\hline SRMR & 0.074 & 0.073 \\
NFI & 0.748 & 0.749 \\
\hline
\end{tabular}

\subsection{Structural Model Assessment}

The structural model assessment of the study involves estimating the relationship among the exogenous (predictor), in this model DM, and endogenous (criterion) variables. The first step of the structural model assessment procedure is the model assessment for collinearity issues. The collinearity assessment was executed using the IBM SPSS software. The results of the collinearity assessment, which is the variance inflation factor or (VIF) estimates are shown in Table 7. All the VIF have values between 1.521 and 2.693, which are below 3.3, which is the threshold for collinearity for variance-based SEM, indicating that there is no problem of collinearity [26]. As collinearity is not an issue at this stage of analysis, the discussion proceeds to structural model assessment.

Table 7 VIF for DM, SI, and SP

\begin{tabular}{|l|l|l|}
\hline DM & SI & SP \\
\hline 2.693 & 1.521 & 1.691 \\
\hline
\end{tabular}

Figure 2 shows the moderation effect of SI on the EDM-SP relationship. While Table 8 lists the summary of results for the structural model assessment for the moderated $\mathrm{DM} \rightarrow \mathrm{SP}(\mathrm{SI} * \mathrm{DM} \rightarrow \mathrm{SP})$ relationship.

The strength of $\mathrm{DM} \rightarrow \mathrm{SP}$ relationship is given as 0.504 which can be considered as strong as it is higher than the recommended value of 0.20 [15]. The empirical $t$ statistics for this relationship is given as 6.447 , which is higher than the critical $t$ value at $1 \%$ level of confidence. Thus, $\mathrm{H}_{1}$ which says that EDM is positively related to $\mathrm{SP}$, is supported.

Whereas the strength of $\mathrm{SI}{ }^{*} \mathrm{DM} \rightarrow \mathrm{SP}$ relationship is given as -0.047 which says that the interaction effect of $\mathrm{SI}$ on $\mathrm{DM} \rightarrow \mathrm{SP}$ relationship is negative. A one standard deviation point increase in SI will lead to 0.047 reduction in SP. The effect of $\mathrm{SI}^{*} \mathrm{DM} \rightarrow \mathrm{SP}$ can be considered as small [15]. The empirical $t$ statistics for the $\mathrm{SI}{ }^{*} \mathrm{DM} \rightarrow \mathrm{SP}$ is given at 0.406 . This value is lower than the critical $t$ value at $10 \%$ level of confidence. Thus, the study does not find that the moderated relationship between EDM and SP as significantly different from 0 at any level of confidence.

The second analysis is the coefficient of determination $R^{2}$ for the predictive accuracy of the model. In the case of the moderated effect of SI on the $\mathrm{EDM}$ and SP relationship $(\mathrm{SI} * \mathrm{DM} \rightarrow \mathrm{SP})$, the $R^{2}$ value is 0.433 , which indicates that together, EDM, and SI can explain $43.3 \%$ of the variation in SP. The explanatory power of the model is slightly increased from $42.9 \%$ to $43.3 \%$ with introduction of the moderator variable of SI.

Table 6. GoF assessment for SI*DM $\rightarrow \mathrm{SP}$ 
Figure 2 Moderation effect of SI on DM $\rightarrow$ SP relationship

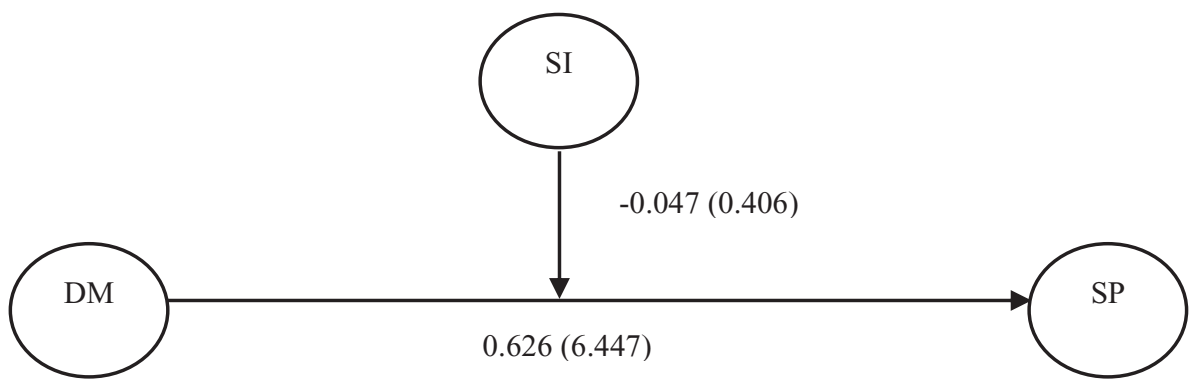

Table 8 Summary of structural model assessment for SI*DM $\rightarrow$ SP relationship

\begin{tabular}{lccccccc}
\hline \multicolumn{1}{c}{ Hypothesis } & Std $\boldsymbol{\beta}$ & $\mathbf{S E}$ & t-value & $\boldsymbol{R}^{2}$ & $\boldsymbol{f}^{2}$ & $\boldsymbol{Q}^{2}$ & Decision \\
\hline $\mathrm{H}_{1}$ : Direct effect of DM $\rightarrow \mathrm{SP}$ & 0.504 & 0.099 & $6.447 * * *$ & 0.429 & 0.245 & 0.159 & Supported \\
$\mathrm{H}_{2}$ : Moderation effect of SI on DM $\rightarrow \mathrm{SP}$ & -0.047 & 0.008 & 0.406 & 0.433 & 0.006 & 0.250 & Not Supported \\
\hline
\end{tabular}

Note: $* * * p<0.01$

Additionally, the $f^{2}$ and $Q^{2}$ analyses were also adopted in evaluating the model's predictive ability. The effect size for the $R^{2}$ of DM is 0.245 , indicating that DM has a moderate effect in producing the $R^{2}$ for SP. While the $f^{2}$ value for SI*DM is 0.006 indicating that $\mathrm{SI}{ }^{*} \mathrm{DM}$ has a very small effect in producing the $R^{2}$ of SP, which indicates that the moderation effect of SI*DM does have much effect in producing the $R^{2}$ for SP. While the $Q^{2}$ analysis is for the predictive relevance of the model, the $Q^{2}$ value for the model is 0.250 (more than zero), which indicates that the overall model has a medium predictive relevance and validity for SP construct [15].

However, authors have argued that in the case of conditional effect analysis (moderation effect), the region of significance for the moderation effect is of more importance [27]. Thus, the analysis continues with examination of the region of significance for the moderation effect. From Figure 3, level of SI has an influence on SP but not on the strength of EDM influence on SP. The higher the level of SI, the higher is the level of SP, which is illustrated by the difference between the dotted line and the continuous line.

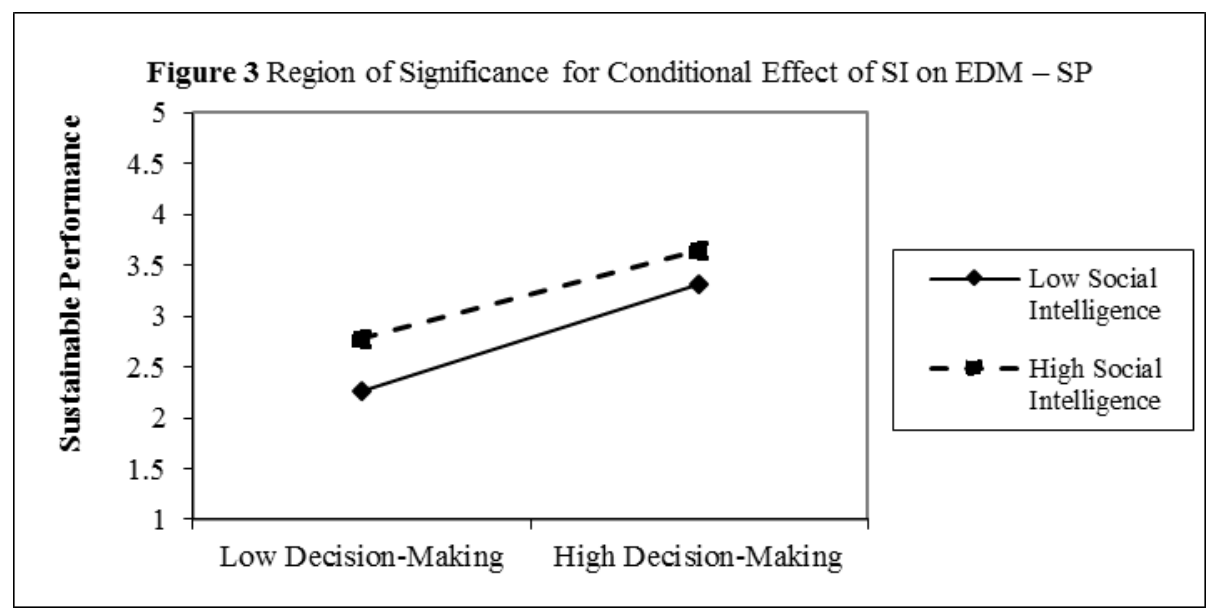

\section{Conclusion and Recommendations}

The study extends the understanding of the effects of EDM, and SI level of the entrepreneurs on the SP of SMEs. The resource-based view (RBV) is adopted as the underlying theory of our hypothesis development. The results reveals that although EDM has a positive influence on SP, it is causation, rather than effectuation that is the significant predictor, which contradicts prior studies [28, 2]. However, the moderated influence of SI on the EDM-SP relationship was not found to be significant. The exploratory study has proposed operationalization and measurement for the constructs of SP based on adoption and adaptation of previously validated measures available in entrepreneurship literature.

\section{Reference}

1. B. Cohen, B. Smith, R. Mitchell. Bus. Strtg. \& Envr. 19(2), 107-119 (2008)

2. S, Read, M. Song, W, Smit. J. Bus. Vent. 24(6), 573-587 ((2009)

3. D, Goleman, HBR. 86(9), 74-81 (2008) 
4. V. Parida, N.M. George, T. Lahti. J. Bus. Rsch. 69(11), 4815-4819 (2016)

5. J.F. Kihlstrom, N. Cantor. Hndbk. Intel. 359-379 (2000)

6. E.D. Heggestad. J. Pers. Assm. 90(1), 102-104 (2008)

7. D. Silvera, M. Martinussen, T.I. Dahl. Scnd. J. Psy. 42(4), 313-319 (2001)

8. R.E. Boyatzis, D. Good, R. Massa. J. Ledrsh. \& Org. Std. 19(2), 191-201 (2012)

9. J. Hartog, M. van Praag, J. van der Sluis. J. Eco. \& Mgt. Strtg. 19(4), 947-989 (2010)

10. P.J. Richard, T.M. Devinney, G.S. Yip, G. Johnson. J. Mgmt. 35(3), 718-804 (2009)

11. J. van der Sluis, M. van Praag, W. Vijverberg. WBER. 19(2), 225-261 (2005)

12. P. Garengo, S. Biazzo, U.S. Bititci. Int. J. Mgmt. Rev. 7(1), 25-47 (2005)

13. NSDC. SME Annual Report 2014/15 (2014)

14. NSDC. SME Annual Report 2011/12 (2011)

15. J.F. Hair, G.T.M. Hult, M. Sarstedt. A primer on partial least squares structural equation modeling (PLS-SEM) (2013)

16. J. Henseler, C.M. Ringle, R.R. Sinkovics. Adv. Int. Mktg. 20(1), 277-319 (2009)

17. J. Hulland. Strtg. Mgt. J. 20(2), 195-204 (1999)
18. B.M. Byrne. Structural equation modeling with AMOS: Basic concepts, applications, and programming. (2016)

19. S.A. Snell, J.W. Dean Jr. T. Acad. Mgt. J. 467-504 (1992)

20. V.E. Vinzi, W.W. Chin, J. Henseler, H. Wang. Handbook of partial least squares: Concepts, methods and applications. (2010)

21. J. Henseler, C.M. Ringle, M. Sarstedt. J. Acad. Mktg. Sci. 43(1), 115-135 (2015)

22. T. Ramayah, J. Cheah, F. Chuah, H. Ting, M.A. Memon. Partial Least-Squares Structural Equation Modeling (PLS-SEM) using SmartPLS 3.0. (2017)

23. R.B. Kline. Convergence of structural equation modeling and multilevel modeling. (2011)

24. L. Hu, P.M. Bentler. SEM: A Multdcpl. J. 691), 155 (1999)

25. J. Lohmoller. Latent variable path modeling with partial least squares. (2013)

26. N. Kock, G.S. Lynn. J. Assoc. Info. Sys. 13(7), 546-580 (2012)

27. A.F. Hayes. Introduction to mediation, moderation, and conditional process analysis: A regressionbased approach. (2008)

28. S. Read, S.D. Sarasvathy. J. Priv. Eqt. 9(1), 45-62 (2005) 